\title{
Sutureless Intrascleral Fixation Of The Carlevale (Soleko) Intraocular Lens In The Correction Of Aphakia. Own Experience
}

\author{
A. Cywinski
}

\begin{abstract}
The article evaluates the functional stability in the eye and the method of intrascleral fixation of spherical or toric, monofocal, intraocular Carlevale lens by Soleko. The procedure was performed in patients requiring correction of aphakia. Haptics of the one-piece lens, due to the T-shape structure are fixed intrasclerally and thus do not require additional fixation sutures. Intrascleral fixation was performed in 14 eyes, in 13 patients, between 2017 and 2019. In one case, a corneal astigmatism correction model (Carlevale toric) was used. Good visual quality and full intraocular stabilization were obtained. Except for transient vitreous haemorrhage, corneal oedema, and elevated intraocular pressure, no other adverse effects were observed, including intraocular displacement, subluxation or rotation of the lens relative to the axis. The normal and stable position of the lens was also obtained in the eyes with accompanying nystagmus.
\end{abstract}

Index Terms - aphakia, eye trauma, intrascleral fixation, astigmatism, nystagmus, Carlevale lens.

\section{INTRODUCTION}

In most cases aphakia is acquired and caused by the removal of the patient's own lens. Removal of the lens in hyperopic eyes and eyes without eye defect causes high hyperopia depriving the patient of useful visual acuity. This condition requires correction to obtain useful visual acuity. In the case of severe myopia, an artificial lens implant is not absolutely required to achieve good vision [1]. In itself aphakia is not a problem when it comes to correcting it. In each case of cataract removal or refractive lens replacement, aphakia is a transient stage which is corrected by introducing an artificial lens, physiologically into the capsule or on its anterior surface. The difficulty in the correction of aphakia increases when the natural support, that is the lens capsule, is damaged. This condition is often observed in the case of eye injuries, including surgical ones, which appear as the main cause of lens damage. Inflammation, some forms of glaucoma (pseudoexfoliation syndrome), some congenital pathologies (Marfan syndrome) are other reasons that may cause the inability to use a lens capsule as support for an artificial lens [2].

Surgeons ophthalmologists have been dealing with the correction of aphakia for many years. At the time when nonfoldable, intraocular acrylic lenses were used, they were introduced into the eye through a large 6-8 $\mathrm{mm}$ incision. The very location of haptic parts in the ciliary sulcus allowed for

Published on July 10, 2020.

A. Cywinski, Silesian Eye Treatment Centre, Poland.

(corresponding e-mail: adamcyw ${ }^{\circledR}$ gmailcom) some intraocular stabilization. At present, in the era of minimizing the incision $(1.8-2.0 \mathrm{~mm})$ through which a foldable lens is introduced, the positioning of the lens in the sulcus is insufficient for its stable fixation. Therefore, many modifications of lens fixation have been introduced. According to Siepser, the number of lens fixation techniques has exceeded 30 combinations in 2011[3]. Transscleral fixation with the application of sutures is the most frequently chosen method [4]. Fixing haptics of the lens to the iris is another method, although its "simpler" version is to use "iris claw" lenses. This lens model is fixed to the iris [5],[7]. Sutureless transscleral fixation of the haptics is just another option. This technique requires the use of a threepiece lens with hard haptic parts and should be the method of choice when a three-piece lens model was originally used [8]. Then the procedure of reimplantation and replacement of the lens with a different model is not required. The use of the above methods may be associated with the occurrence of a number of complications. Rupture of the lens fixation with the application of suture thread is one of the more serious complications observed in the case of transscleral fixation. Re-subluxation of the lens, macular oedema, appearance of the pre-macular membrane, damage to the structure of the iris, disturbance of the pupil function and change in its shape may accompany the technique of fixing haptics to the iris [6],[7]. Techniques of sutureless fixation of haptics in the transscleral tunnel are associated with the risk of complications associated mainly with damage of the haptics. Haptic kink, breakage, dislocation or slippage and less frequently IOL dislocation have been described in literature [8],[9]. Each of the complications lead to a decrease in quality and acuity of vision. Hong et al. showed that the risk of postoperative complications does not exceed $20 \%$ and includes, among others, elevation of IOP, IOL dislocation or irregular pupil. A more serious complication was corneal oedema, requiring DSAEK, which was observed in $7.5 \%$ of a group of 135 eyes. Other complications, observed in less than $10 \%$ of all cases were: vitreous haemorrhage, hyphema, macular oedema and retinal detachment [2]-[9].

The author of this article, using many lens fixation techniques in his practice (except for sutureless, transscleral fixation using three-piece lenses), often observed the abovementioned complications. In the case of late complications, rupture of the fixation thread with subsequent subluxation of the lens was one of the most serious consequences. When using the method of fixation of the lens to the iris, pigment dispersion, irregular pupil and tears of the iris in the sites of fixation of the lens and iridodonesis are the most common complications. 
Nystagmus increases the difficulty of lens fixation and the risk of postoperative complications. Therefore, some techniques, including fixation of the lens to the iris, should not be recommended.

\section{OBJECTIVE}

Evaluation of visual acuity, intraocular stability and the risk of complications in eyes subjected to the correction of aphakia using a one-piece, foldable intraocular Carlevale lens by Soleko. The main reason for choosing this lens model was the lack of support in the eye for an artificial lens and the desire to avoid fixation sutures. The structure of the lens allows for its intrascleral fixation, without the need for additional sutures. Haptics of the Carlevale model are Tshaped (Fig. 1).

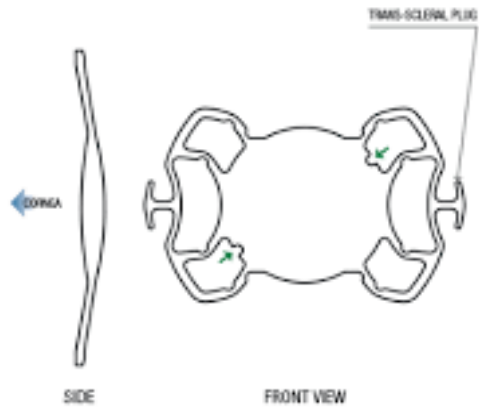

Fig. 1. Carlevale - spherical model by Soleko. T-shaped haptics allows to fixate the lens without additional suture.

Thanks to this structure, the lens gains some fixation in the eye. The flexible lateral elements of the haptic parts rest on the internal structures of the eyeball wall, and thus the lens adapts to the eyes of different sizes. The Carlevale toric model is used when astigmatism correction is needed (Fig. 2).

\section{Real Axis Technology}

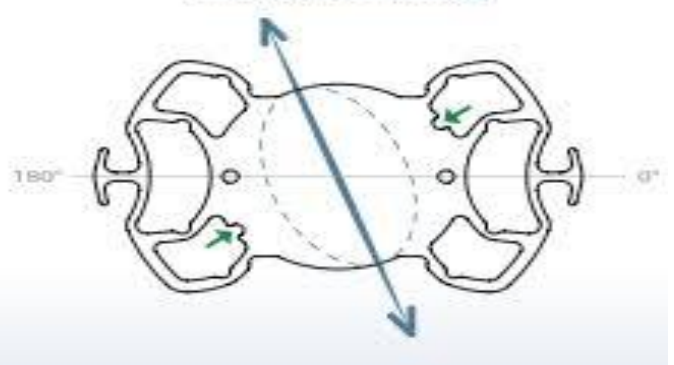

Fig. 2. Carlevale - toric model by Soleko for correction of corneal astigmatism. The lens can be positioned in two axes: $0^{\circ}-180^{\circ}$ or $90^{\circ}-270^{\circ}$.

\section{MATERIAL AND METHOD}

Thirteen people, 14 eyes with confirmed lack of support were subjected to transscleral fixation using the Carlevale lens. All procedures were performed at the private medical centre - Silesian Eye Treatment Centre (Zory, Poland), by one surgeon - Dr. Cywinski.

The implantation stages included 1. Determining the eyeball axis to determine symmetrical locations for transscleral pockets. 2. Creation of transscleral pockets using a crescent knife with a blade diameter of $2.0 \mathrm{~mm}$, with an entrance from the corneal limbus. 3. Making incisions to enter the eye $1.5 \mathrm{~mm}$ from the corneal limbus using a $23 \mathrm{G}$ MVR knife. 4. Insertion of the lens into the anterior chamber through a 2.2-2.4 mm main incision. In the eyes after a posterior vitrectomy procedure, at the beginning of the surgery an irrigation port was inserted into the anterior chamber to maintain constant tension of the eyeball. In the eyes that did not require vitrectomy, viscoelastic fluid was introduced into the anterior chamber before inserting the artificial lens, 5. A two-handed technique of passing the haptic part of the lens through the scleral pocket using $23 \mathrm{G}$ endoforceps or one endoforceps and a hook. 6. Inserting of the haptic part into the scleral pocket, 7. Suturing of the conjunctival wound. All stages of lens fixation were identical for both the spherical and toric lens implantation, with one exception. In the latter case, the corrected astigmatism axis is determined in the first stage. It is possible to choose between $0^{\circ}-180^{\circ}$ or $90^{\circ}-270^{\circ}$ axes. The astigmatism axis appropriate for a given eye is determined by the lens manufacturer, according to the calculation made. Transscleral pockets are formed on the designated axis.

\section{RESUlTS}

The average age of patients was 41 years, in the range from 23 to 82 , of which over $60 \%$ of patients were male. The values of intraocular lenses ranged from 6 to 26 dioptres. In 13 cases (eyes) spherical lenses were used, in one case a toric lens was used. The average follow-up period was 5 months, in the range from 3 to 20 months.

Eye injury was the main cause of aphakia in 10 people (eyes). Intrascleral fixation in both eyes was performed in one patient due to aphakia following congenital cataract surgery. Surgical correction of strabismus and nystagmus were performed in this patient before the lens implantation.

In 8 people (eyes) the lens implant was combined with another surgical procedure. In 4 cases it was the removal of a previously implanted artificial lens due to its subluxation (1 eye), another rupture of the lens fixation thread (1 eye).

In another two patients ( 2 eyes) the removal of the artificial lens was dictated by its opacification (calcification).

In 4 patients, fixation of the lens was combined with iris plastic surgery to reduce pupil diameter.

Before fixation procedure, 8 people (eyes) underwent posterior vitrectomy, which was performed in another centre due to consequences of an injury to the eye. Causes of aphakia and types of surgery performed are listed in Table I.

TABLE I. CAUSES OF APHAKIA AND TyPes OF SURGERY PERFORMED

\begin{tabular}{lc}
\hline \hline Causes and types of procedures performed & $\begin{array}{c}\text { Number of } \\
\text { patients (eyes) }\end{array}$ \\
\hline Aphakia following eye injury & $8(8)$ \\
Aphakia following congenital cataract removal & $1(2)$ \\
$\begin{array}{l}\text { Subluxation, calcification of the artificial lens } \\
\text { Fixation of the Carlevale lens }\end{array}$ & $4(4)$ \\
$\begin{array}{l}\text { Complex procedure - removal of artificial subluxated } \\
\text { lens and Carlevale lens fixation }\end{array}$ & $5(6)$ \\
$\begin{array}{l}\text { Complex procedure - removal of calcified artificial } \\
\text { lens and Carlevale lens fixation }\end{array}$ & $2(2)$ \\
\hline
\end{tabular}




\section{A. Visual acuity.}

A comparative analysis of uncorrected visual acuity (UCVA) and with best-corrected visual acuity (BCVA) was performed in the preoperative period and 5 months after surgery. Obtained values of far and near vision were presented in the form of graphs. Far visual acuity values were tested using log MAR charts (Graph1), near visual acuity values - using Snellen charts (Graph2).

\section{Visual acuity to far distances before and after} surgery

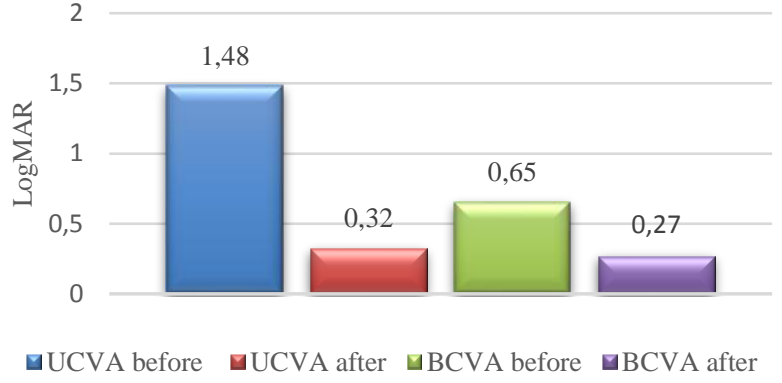

Graph 1 . Visual acuity values to far distances before and after surgery

BCVA to near distances before and after surgery

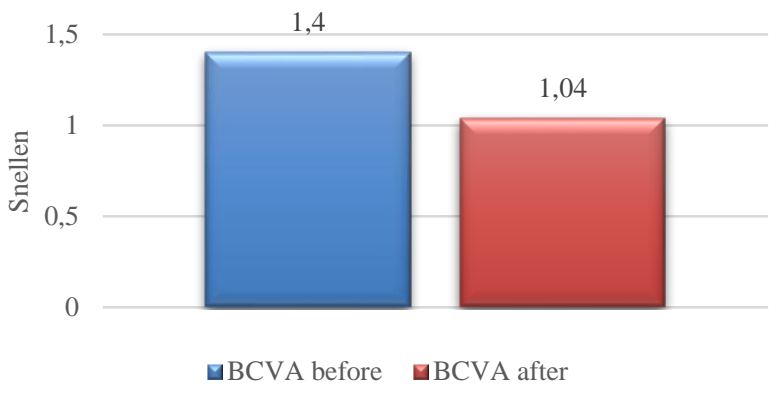

Graph 2. BCVA values to near distances before and after surgery.

Visual acuity improved in every case except for a patient who had a post-injury full thickness, large scar of the cornea. This patient underwent a complex procedure combining lens fixation and iris plastic surgery to reduce pupil diameter. After the procedure, a progressive increase in oedema and corneal haze were observed. The patient was qualified for corneal transplantation.

In the case of the patient with nystagmus who had intrascleral fixation surgery in both eyes, both far and near visual acuity improved insignificantly.

Toric lens implantation was performed in a 28 -year-old patient with Marfan syndrome, in whom the lens fixation thread was ruptured and subsequently subluxated as a result of a blunt trauma of the eye. The subluxated lens was removed and a Carlevale toric lens was implanted at the same time. A reduction in astigmatism was obtained from 2.5 Dcyl to 0.25 Dcyl. Distance BCVA improved from the value of 0.4 before surgery to $0.3,5$ months after.
The best results of UCVA improvement were obtained in aphakic eyes, which seems logical. Aphakia in eyes without eye defect and hyperopic eyes generates high hyperopia. Although the artificial lens value calculation was aimed at obtaining emmetropia after surgery, a residual postoperative defect was observed in all but one case. In a patient with a subluxated, rigid acrylic lens implanted about 20 years earlier, it was not possible to centre the lens despite three attempts made in other centres. Due to the inability to remove the entire lens (fusion of the haptics with the eye tissues), only its optical part was removed cutting off the haptics. Far UCVA has significantly improved from preoperative value of 1.8 to 0.1 (LogMAR). A similar distance UCVA value was obtained in a 38-year-old man in a post-injury eye in which the patient's damaged lens was removed and posterior vitrectomy was performed. Despite the presence of a small paracentral corneal scar, UCVA reached 0.0 fere (LogMAR) for distance vision, and D-0.5 for near vision examined using Snellen charts. In the above case, fixation of the lens was combined with surgical reduction of pupil diameter.

In the examined group of patients, the value of postoperative residual defect did not exceed 1.25Dsph and 1.25Dcyl. In the case of procedures combining lens fixation with reduction of the pupil diameter, a reduction in photophobia and glare was additionally achieved. All stages of Carlevale intrascleral fixation, in addition to reducing the pupil diameter, are shown in the video below:

https://medtube.pl/okulistyka/filmy-medyczne/26579. intrascleral-fixation-of-carlevale-combined-with-decreaseof-the-pupil-size.

Except for the case eligible for corneal transplant, each of the patients who underwent surgery expressed their satisfaction with it. In addition, to the question asked whether patients would choose to have the above procedure performed again, each of them answered affirmatively.

\section{B. Complications}

Intraoperative complications include mainly bleeding to the anterior chamber and the vitreous. Postoperatively, they include the presence of dispersed blood, mainly in the vitreous chamber, despite its removal at the end of the procedure. This complication was observed in 10 out of 14 eyes. Blood was usually absorbed within 1-2 postoperative weeks. Transient corneal oedema is another complication observed in 9/14 eyes, although the average endothelial cell density exceeded $1,600 / \mathrm{mm} 2$. The exception was a patient with a huge post-injury corneal scar. A transient increase in intraocular pressure, lasting about 7-14 days, was observed in 9 eyes. This condition normalized after applying antiglaucomatous eye drops. In 3 cases in which postoperative pressure reached values above $35 \mathrm{mmHg}$, additionally dorzolamide was administered orally.

\section{DISCUSSION}

Intrascleral fixation of the Carlevale lens is another option to fix an artificial lens in the eyes without capsula support Considering the follow-up results described in the available publications, the procedure using this lens is an effective and safe procedure [10]-[12]. It seems that the risk of lens subluxation is practically impossible, which is guaranteed 
by the structure of the haptics of the lens and thus its fixation method.

Transient postoperative complications were observed in most patients. These lesions disappeared quite quickly, within 2 weeks after surgery. The percentage of complications observed in the study group was much higher compared to the results obtained by other authors [10]-[12]. This may be related to the complexity of procedures performed, during which, in addition to removing previous implants, iris procedures were performed, mainly aimed at reducing pupil diameter.

The insufficient follow-up period does not allow for an assessment of late possible adverse effects after implantation of this lens model. The benefits of using the Carlevale lens primarily include no risk of progressive iris damage, as seen with the lens fixed to the iris. Avoiding damage to the haptics observed when using a three-piece lens, which, however, are not adapted to additional surgical manoeuvres, and the already mentioned lack of risk of rupture of the lens fixation thread are further benefits of using this model of an intraocular lens.

\section{CONCLUSIONS}

Intrascleral fixation of the Carlevale lens by Soleko used in the correction of aphakia is a safe method that allows for the achievement of the intended, predictable effect of improving the quality of vision in eyes in which there is no support for an artificial lens in the form of a lens capsule.

The appearance of transient postoperative complications does not adversely affect the permanent effect of the procedure and no additional procedures are required to remove them. The structure of the haptic parts of the lens allows for its full stabilization, even in eyes with accompanying nystagmus.

\section{REFERENCES}

[1] T. Ochi, A. Gon, Y. Kora, K. Kawai, Y. Fukado. Intraocular Lens Implantation and High Myopia Cataract Refract Surg. 1988 Jul;14(4):403-8.

[2] A. Hong, J. Kuryan, E. Wu, D. Ritterband, J. Seedor. Clinical Outcomes and Complications of Iris Sutured Posterior Chamber Intraocular Lens Implants. Investigative Ophthalmology \& Visual Science July 2017, Vol.58, 3530-3536.

[3] SB. Siepser New method of transscleral fixation an alternative for securing IOLs Ocular Surgery News U.S. Edition, April 25, 2011,

[4] E. Nudleman, Y. Yonekawa , JL. Prenner. Sutureless Transscleral Fixation of Secondary Intraocular Lenses. Curr Opin Ophthalmol. 2018 May;29(3):210-216.

[5] AM. Bedda, HF. Goweini, AM. Abdelhadi, AM. Elhady. Evaluation of Suturless Scleral Fixation with Posterior Chamber Foldable Intraocular Lens Implantation. Int J Ophthalmol. 2019 Aug 18;12(8):1283-1289.

[6] J. Wu, Liang Guanlu; Zheng Qianyin; Li Shuyi; He Fengying; Liu Jian; $\mathrm{Xu}$ Wen. Iris-Claw Intraocular Lens and Scleral-Fixated Posterior Chamber Intraocular Lens Implantations in Correcting Aphakia: A Meta-Analysis Investigative Ophthalmology \& Visual Science July 2017, Vol.58, 3530-3536.

[7] El-Saadany Abdel-Khalik I, Khairy Hany A, Z. Rajab Ghada, Shebl Fayed Ahmed M. Menoufia Evaluation of aphakic iris-claw intraocular lens implantation Medical Journal 2019, Vol.32, (2): 690697

[8] S. Ni, W. Wang, X. Chen et al. Clinical observation of a novel technique: transscleral suture fixation of a foldable 3-looped haptics one-piece posterior chamber intraocular lens implantation through scleral pockets with intact conjunctiva. BMC Ophthalmol 19, 105 (2019).
[9] D. Dzhaber, OM Mustafa, J. Tian, JT Cox, YJ. Daoud. Eye Outcomes and complications of iris-fixated intraocular lenses in cases with inadequate capsular support and complex ophthalmic history. (London, England), 03 Jan 2020.

[10] F. Barca, T. Caporossi, L. de Angelis, F. Giansanti, A. Savastano, L. Di Leo, S. Rizzo. Trans-scleral plugs fixated IOL: a new paradigm for sutureless scleral fixation. Journal of Cataract \& Refractive Surgery: May 2020 - Volume 46 - Issue 5 - p 716-720.

[11] C. Veronese, C Maiolo, GW Armstrong et al. New surgical approach for sutureless scleral fixation. Eur J Ophthalmol. 2020;30(3):612-615.

[12] M. Petrelli, L. Schmutz, E. Gkaragkani, K. Droutsas, G. Kymionis. Simultaneous Penetrating Keratoplasty and Implantation of a New Scleral-Fixated, Sutureless, Posterior Chamber Intraocular Lens (Soleko, Carlevale), Cornea: May 21, 2020 - Volume Publish Ahead of Print - Issue.

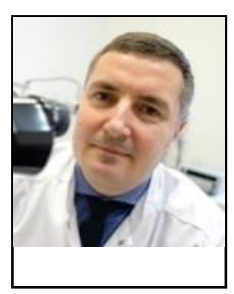

Adam Cywinski, date of birth 22.09.1967. Medical Director and founder of private medical center, Silesian Eye Treatment Centre, Zory, Poland. Consultant of posterior segment diseases. He graduated Silesian Medical Academy in Katowice and gained medical degree in the biggest Clinic of Ophthalmology, also in Katowice. He doctorated in surgical topic "Posterior vitrectomy with gas endotamponade and ILM peeling in IV stage macular hole, in 2003. As a surgeon he made a few thousands posterior vitrectomy procedures, including macular diseases, retinal detachment, diabetic and posttraumatic cases. His next interest is anterior segment surgery with special focus on reconstruction of the iris. His everyday job includes cataract surgery of complicated cases and surgery with premium class lenses. Dr Cywinski is a member of PTO, AAO, ESCRS, Euretina. 